\title{
TRES NUEVAS ESPECIES DEL GÉNERO APHIS LINNAEUS (HEMIPTERA, APHIDIDAE) VIVIENDO SOBRE VERBENÁCEAS (VERBENACEAE) EN LA ARGENTINA
}

\author{
Sandra González Rodríguez ${ }^{1}$ M. Pilar Mier Durante ${ }^{2}$ Jaime Ortego $^{3}$ \& Juan M. Nieto Nafría ${ }^{4}$ \\ 'Departamento de Biodiversidad y Gestión Ambiental, área de Zoología. Universidad de León. 24071 León (España). \\ https://orcid.org/0000-0002-0301-6740-sgonzr02@estudiantes.unileon.es \\ ${ }^{2}$ Departamento de Biodiversidad y Gestión Ambiental, área de Zoología. Universidad de León. 24071 León (España). \\ https://orcid.org/0000-0001-7834-1778 - mpmied@unileon.es \\ ${ }^{3}$ Instituto Nacional de Tecnología Agropecuaria (INTA). Estación Experimental Agropecuaria Mendoza. San Martín, 3853.5507 \\ Luján de Cuyo (Mendoza, Argentina). https://orcid.org/0000-0001-9738-7085 - ortegojaime@yahoo.com.ar \\ ${ }^{4}$ Departamento de Biodiversidad y Gestión Ambiental, área de Zoología. Universidad de León. 24071 León (España). \\ https://orcid.org/0000-0001-6714-2415 - jmnien@unileon.es
}

\section{RESUMEN}

Se describen tres especies del género Aphis Linnaeus (Hemiptera, Aphididae, Aphidinae) a partir de hembras vivíparas ápteras y aladas recogidas sobre especies de Junellia, Lippia y Mulguraea (Verbenaceae) en localidades de la Argentina, respectivamente: Aphis junelliae González Rodríguez \& Nieto Nafría sp. n., Aphis lippiae Ortego \& Nieto Nafría sp. n., Aphis mulguraeae Nieto Nafría \& Mier Durante sp. n. Se exponen las características que permiten diferenciar las nuevas especies entre sí y de las restantes especies de Aphis conocidas en América del Sur.

$$
\text { urn:Isid:zoobank.org:pub:A365C410-F84A-40DE-8C5B-13C2FA768EFC }
$$

Palabras clave: Pulgones; áfidos; especies nuevas; Aphis; Verbenaceae, Junellia; Lippia; Mulguraea.

\section{ABSTRACT}

Three new species of genus Aphis Linnaeus (Hemiptera, Aphididae) living on verbenaceous plants (Verbenaceae) in Argentina

Three new species belonging to the genus Aphis Linnaeus (Hemiptera, Aphididae, Aphidinae) are described from apterous and alate viviparous females collected on species of Junellia, Lippia and Mulguraea (Verbenaceae) in localities of Argentina; they respectively are: Aphis junelliae González Rodríguez \& Nieto Nafría sp. n., Aphis lippiae Ortego \& Nieto Nafría sp. n., Aphis mulguraeae Nieto Nafría \& Mier Durante sp. n. Features that distinguish the new species between them and from other species of Aphis known in South America are exposed.

Key words: Aphids; new species; Aphis; Verbenaceae, Junellia; Lippia; Mulguraea.

Recibido/Received: 27/11/2017; Aceptado/Accepted: 18/05/2018; Publicado en línea/Published online: 25/06/2018

Cómo citar este artículo/Citation: González Rodríguez, S., Mier Durante, M. P., Ortego, J. \& Nieto Nafría, J. M. 2018. Tres nuevas especies del género Aphis Linnaeus (Hemiptera, Aphididae) viviendo sobre verbenáceas (Verbenaceae) en la Argentina. Grael/sia, 74(1): e070. https://doi.org/10.3989/graellsia.2018.v74.194

Copyright: (C) 2018 SAM \& CSIC. This is an open-access article distributed under the terms of the Creative Commons Attribution 4.0 International (CC BY 4.0) License. 


\section{Introducción}

El género Aphis Linnaeus, 1758 es el que tiene mayor número de especies válidas de la familia Aphididae (Hemiptera); según Favret (2017) el género incluye 580 sin tener en cuenta las incluidas en el subgénero Iowana Hottes, 1954, taxón al que otros autores (por ejemplo Blackman \& Eastop, 2017) asignan rango de género. De 535 de ellas se conocen con certeza sus plantas hospedadoras (Blackman \& Eastop, 2017). En América del Sur se han citado 63 especies del género (López Ciruelos et al., 2017), de todas ellas sabemos cuáles son sus plantas hospedadoras, o al menos alguna de ellas en las especies cuya monofagia no está confirmada.

La familia Verbenaceae incluye 34 géneros con algo más de 1000 especies, y está distribuida principalmente en las regiones tropicales y templadas del hemisferio sur. La presencia de la familia en la Argentina es importante, con 26 géneros y 180 especies (The Plant List, 2013; Múlgura de Romero et al., 2012).

Las especies de pulgones que se han citado en el Mundo sobre Verbenaceae, concretamente sobre 15 de sus géneros, son en su mayoría especies polífagas y cosmopolitas o de amplia distribución (Blackman \& Eastop, 2017). Nueve de esas especies pertenecen al género Aphis, a saber: (1) A. matilei Nieto Nafría, Ortego \& Mier Durante, 2000, que vive sobre Junellia spathulata var. glauca (Verbena glauca en la descripción de la especie de pulgón [Nieto Nafría et al., 2000]) y es conocida solamente en Argentina; (2) A. mamonthovae Davletshina, 1964, que vive sobre especies de Verbena, principalmente $V$. officinalis, y se ha citado en varios países desde el sudoeste de Europa (Portugal, España e Italia) hasta el norte de Etiopía y norte de la India (García Prieto \& Nieto Nafría, 2005; Barbagallo \& Massimino Cocuzza, 2014; Blackman \& Eastop, 2017); y (3) A. (Toxoptera) aurantii Boyer de Fonscolombe, 1941, A. craccivora Koch, 1854, A. fabae Scopoli, 1763, A. gossypii Glover, 1877, A. nasturtii Kaltenbach, 1843, A. solanella Theobald, 1914) y A. spiraecola Patch, 1914 (Blackman \& Eastop, 2017) que son polífagas en distinto grado y presentan amplias distribuciones.

En la colección de la Universidad de León se conservan seis muestras de Aphis recogidas sobre verbenáceas en las provincias argentinas de Córdoba, La Rioja, Mendoza y San Juan; su estudio permite describir tres nuevas especies.

\section{Material y métodos}

Los datos de colecta de las especies nuevas se exponen en el apartado dedicado a cada una de ellas. Los ejemplares se montaron en preparación microscópica y sus datos métricos se han tomado de acuerdo con lo establecido por Nieto Nafría \& Mier Durante (1998).
Los dibujos han sido realizados por S. González Rodríguez utilizando una cámara clara colocada en un microscopio de campo claro Laborlux X de Leitz: Las microfotografías fueron tomadas por Sara I. López Ciruelos utilizando un microscopio BX61 con cámara digital DP70 y un sistema informático DP Manager Software version 2.1.1.163 de Olympus.

\section{Resultados y discusión}

\author{
Aphis junelliae González Rodríguez \& Nieto \\ Nafría, sp. n. \\ urn:1sid:zoobank.org:act:83BC83DE-CD32- \\ 421F-BAED-8AD6B1403433
}

Figs. 1A-E, 2A-B, Tabla 1

\section{Material tipo}

Holotipo: hembra vivípara áptera (número 2 de la serie de medidas), ARGENTINA, provincia de San Juan, $32 \mathrm{~km}$ oeste de Baños de Pismanta [aproximadamente $30^{\circ} 20^{\prime} \mathrm{S}, 6^{\circ} 23^{\prime} \mathrm{W}, 2300 \mathrm{~m}$ ], 24-XI-2002, sobre Junellia crithmifolia, Mier Durante, Nieto Nafría y Ortego leg., colección de la Universidad de León (León, España).

Paratipos: 11 hembras vivíparas ápteras y 1 hembra vivípara alada, recogidas junto al holotipo, colecciones de la Universidad de León (León, España) y de J. Ortego (Mendoza, Argentina).

Hembras vivíparas ápteras (Figs. 1A, 1D, 1E, 2A): A partir de 12 especímenes. De 0,90 a 1,21 mm de longitud corporal incluyendo la cola. Otras características métricas en Tabla 1 . En vida de color verde con una mancha rojiza entre los cornículos, éstos son marrones, y la cola parduzca. Una vez preparadas son pálidas en general, con algunas partes del cuerpo más o menos pigmentadas (ver más adelante). Setas de las antenas y patas así como las dorsales del cuerpo robustas y puntiagudas. Dorso de la cabeza liso y algo pigmentado, aunque menos que el clípeo, que es pardo, y que las láminas mandibulares y maxilares. Frente casi plana. Antenas con seis o cinco artejos (incluso en un mismo ejemplar), estando el artejo I, el último y gran parte del penúltimo, algo pigmentados; artejo III de las antenas hexasegmentadas con 3-5 setas. Rostro largo, su extremo alcanza los primeros segmentos del abdomen. Artejo apical del rostro relativamente estrecho, de bordes ligeramente cóncavos y con pigmentación similar a la del penúltimo artejo y a la del dorso de la cabeza; con dos setas complementarias muy largas. Segmentos torácicos con escleritos marginales de límites muy mal definidos y muy poco pigmentados. Papilas marginales del protórax hemisféricas, algo menos voluminosas que el triomatidio y muy pálidas. Coxas tan pigmentadas como el dorso de la cabeza y como una pequeña porción dorsoapical de los fémures (al menos de los posteriores) y algo menos que los 


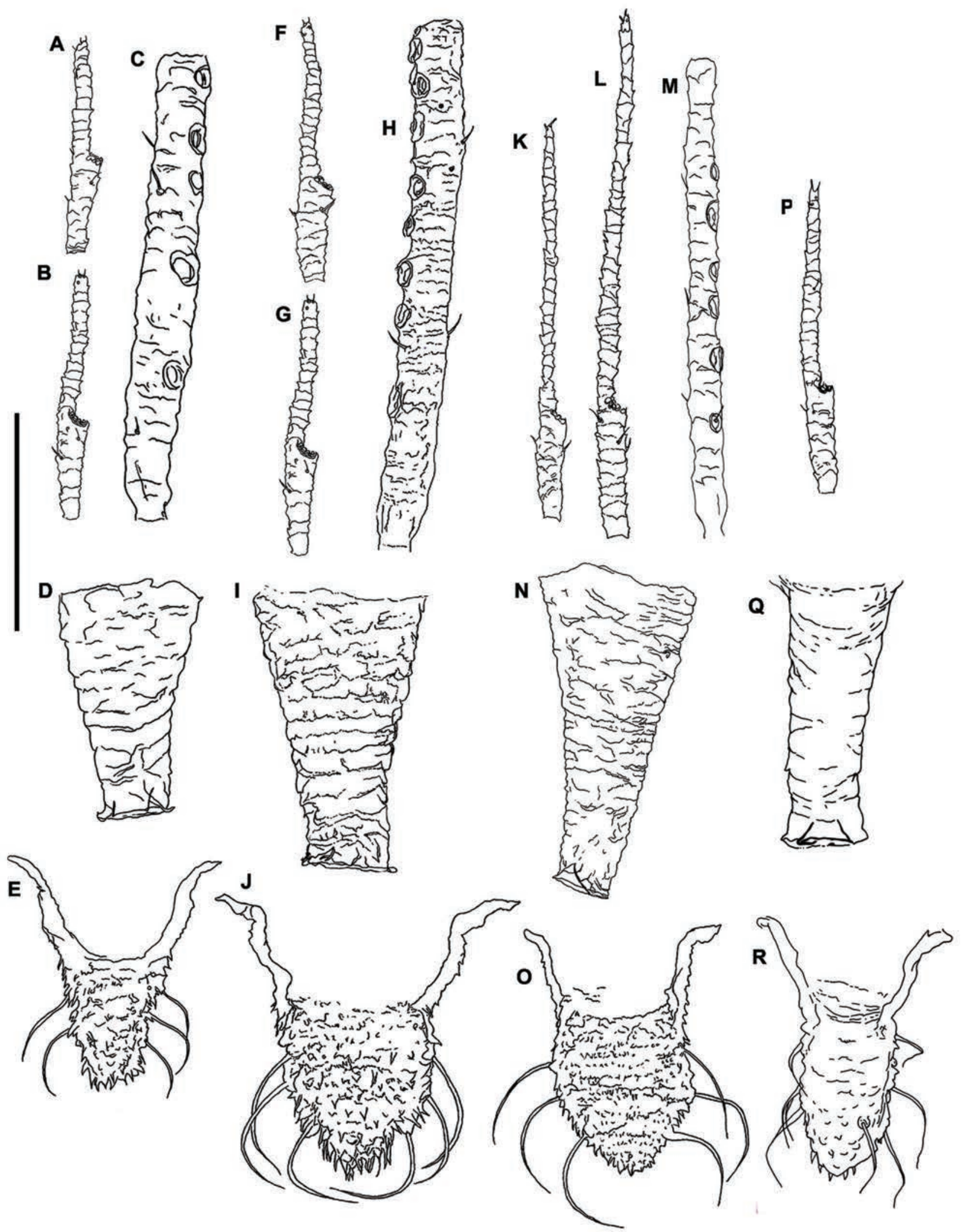

Fig. 1.- A-E Aphis junelliae sp. n.; F-J, Aphis lippiae sp. n.; K-O, Aphis mulguraeae sp. n.; P-R, Aphis matilei; A, D-F, I-K, $\mathrm{N}-\mathrm{R}$, hembra vivípara áptera; $\mathrm{B}-\mathrm{C}, \mathrm{G}-\mathrm{H}, \mathrm{L}-\mathrm{M}$, hembra vivípara alada; $\mathrm{A}-\mathrm{B}, \mathrm{F}-\mathrm{G}, \mathrm{K}-\mathrm{L}, \mathrm{P}$, artejo antenal VI; C, H, M, artejo antenal III; D, I, N, Q, cornículo; E, J, O, R, cola. Las pigmentaciones han sido suprimidas. Escala: 0,1 mm.

Fig. 1.- A-E Aphis junelliae sp. n.; F-J, Aphis lippiae sp. n.; K-O, Aphis mulguraeae sp. n.; P-R, Aphis matilei; A, D-F, I-K, $\mathrm{N}-\mathrm{R}$, apterous viviparous female; $\mathrm{B}-\mathrm{C}, \mathrm{G}-\mathrm{H}, \mathrm{L}-\mathrm{M}$, alate viviparous female; $\mathrm{A}-\mathrm{B}, \mathrm{F}-\mathrm{G}, \mathrm{K}-\mathrm{L}, \mathrm{P}$, antennal segment $\mathrm{Vl} ; \mathrm{C}, \mathrm{H}, \mathrm{M}$, antennal segment III; D, I, N, Q, siphunculus; E, J, O, R, cauda. Pigmentation has been supressed in all draws. Scale bar: $0.1 \mathrm{~mm}$. 


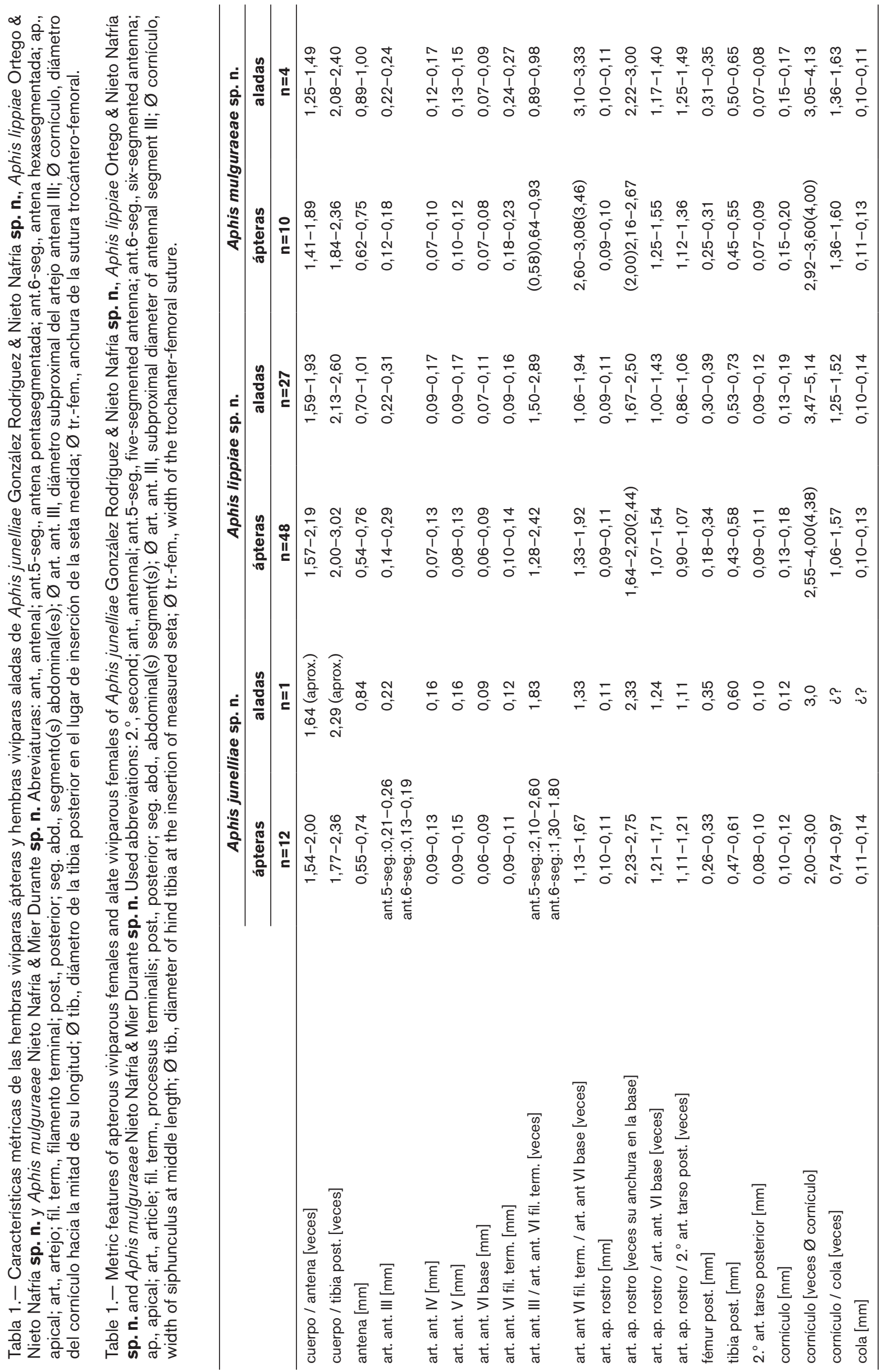




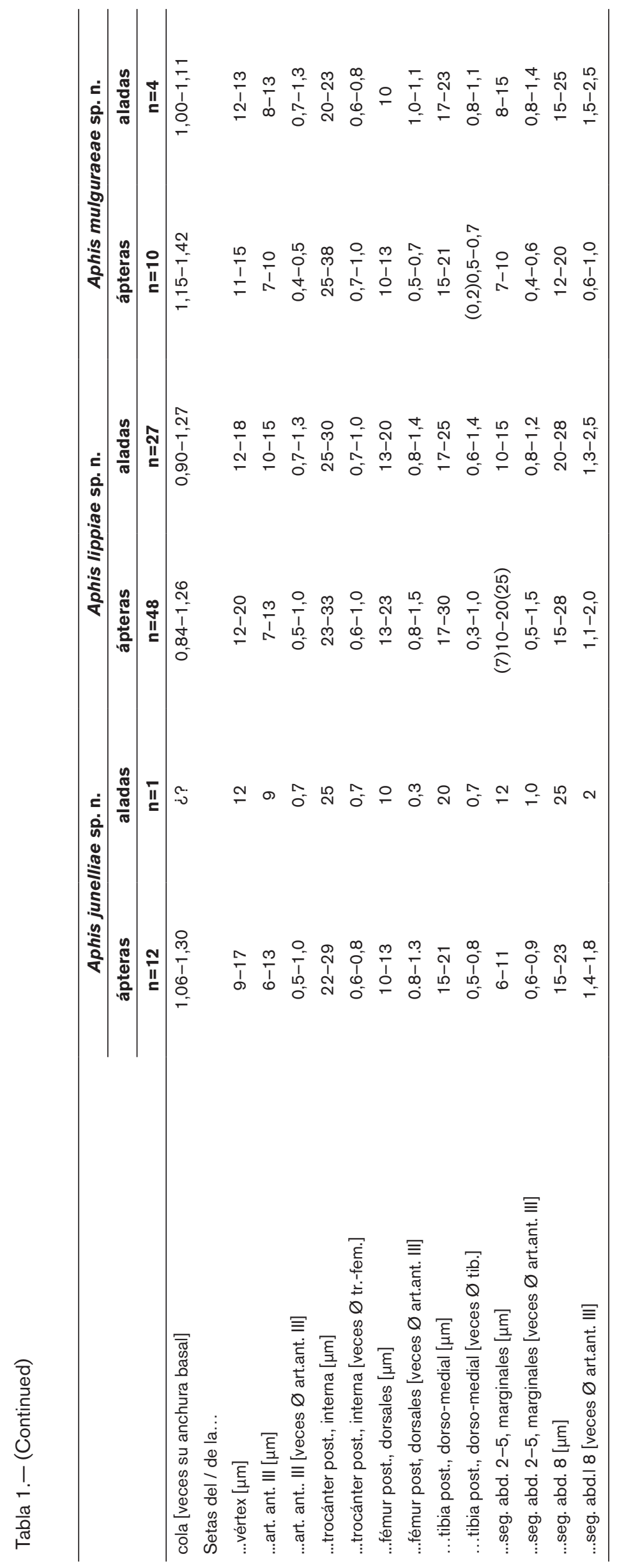


tarsos y una porción distal de las tibias. Fórmula tarsal 3.3.3. Esclerotización dorsoabdominal segmentaria variada: en la zona espinal de los segmentos 2-6 de dos ejemplares hay celdillas pigmentadas que llegan a fusionarse en parches transversales; en dos ejemplares también hay pequeños escleritos setíferos en el segmento 8; escleritos estigmáticos pequeños $\mathrm{y}$ tenuemente pigmentados. Escleritos intersegmentarios poco marcados y a veces inconspicuos. Papilas marginales de los segmentos abdominales 1 y 7 , pálidas y menos voluminosas que las del protórax; segmentos abdominales 2-6 sin ellas. Cornículos pardos (más o menos tan pigmentados como el clípeo), cortos, troncocónicos, con ornamentación de líneas transversales de espínulas poco marcadas, y con reborde muy poco definido y pálido. Segmento abdominal 8 con 2-4 setas. Placas genital y anal algo pigmentadas; aquélla con 2(3) setas discales y 6-9 marginales. Cola digitiforme, aunque la constricción proximal no está muy marcada, poco pigmentada y con 4-7 setas caudales largas y curvadas.

Hembras vivíparas aladas (Figs. 1B, 1C, 2B): A partir de 1 espécimen, de algo más de $1,37 \mathrm{~mm}$ de

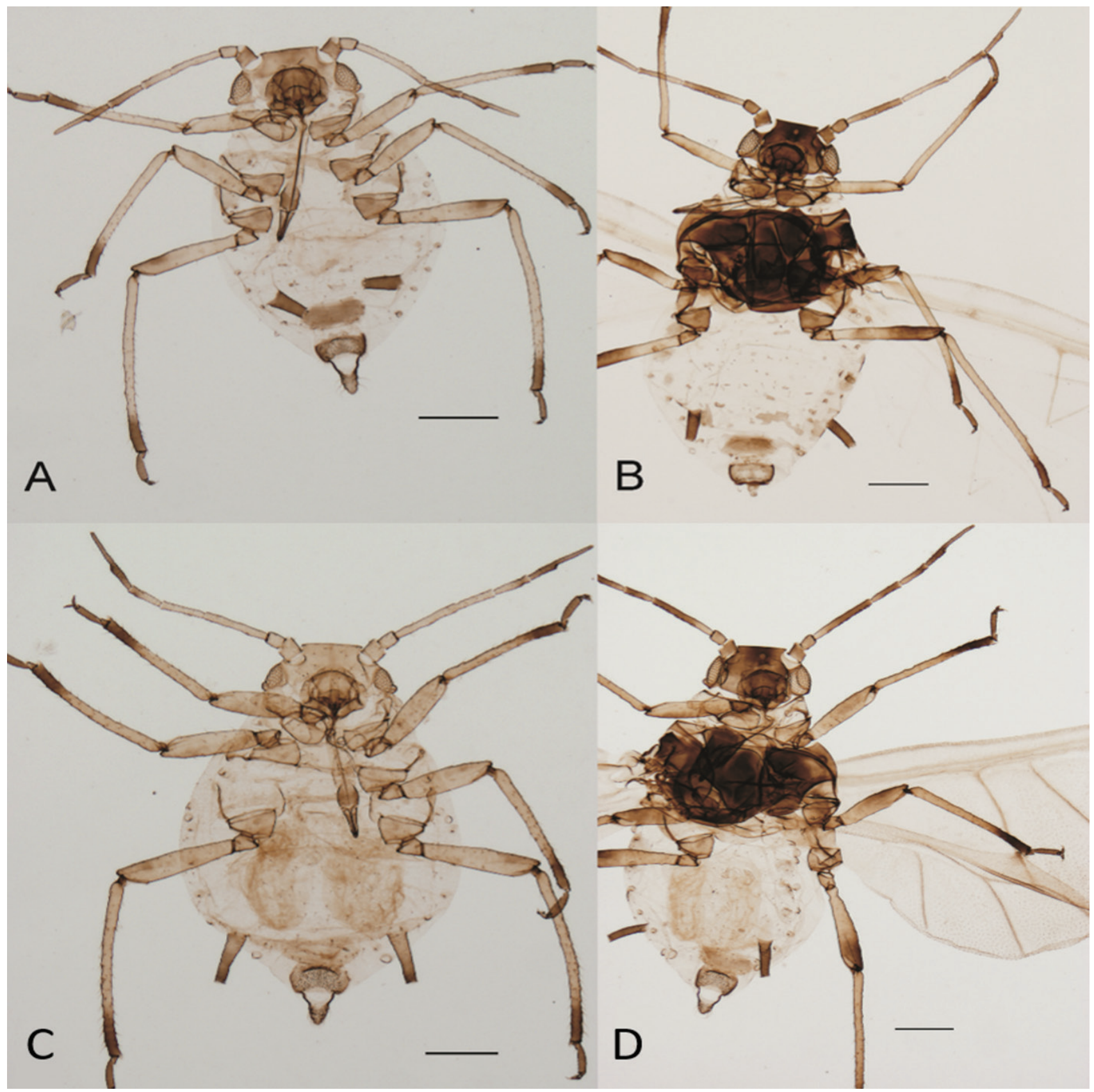

Fig. 2.- A-B, Aphis junelliae González Rodríguez \& Nieto Nafría, sp. n.; C-D, Aphis lippiae Ortego \& Nieto Nafría, sp. n.; A, C, hembra vivípara áptera; B, D, hembra vivípara alada. Escalas: $200 \mu \mathrm{m}$.

Fig. 2.- A-B, Aphis junelliae González Rodríguez \& Nieto Nafría, sp. n.; C-D, Aphis lippiae Ortego \& Nieto Nafría, sp. n.; A, C, apterous viviparous female; B, D, alate viviparous female. Scale bars: $200 \mu \mathrm{m}$. 


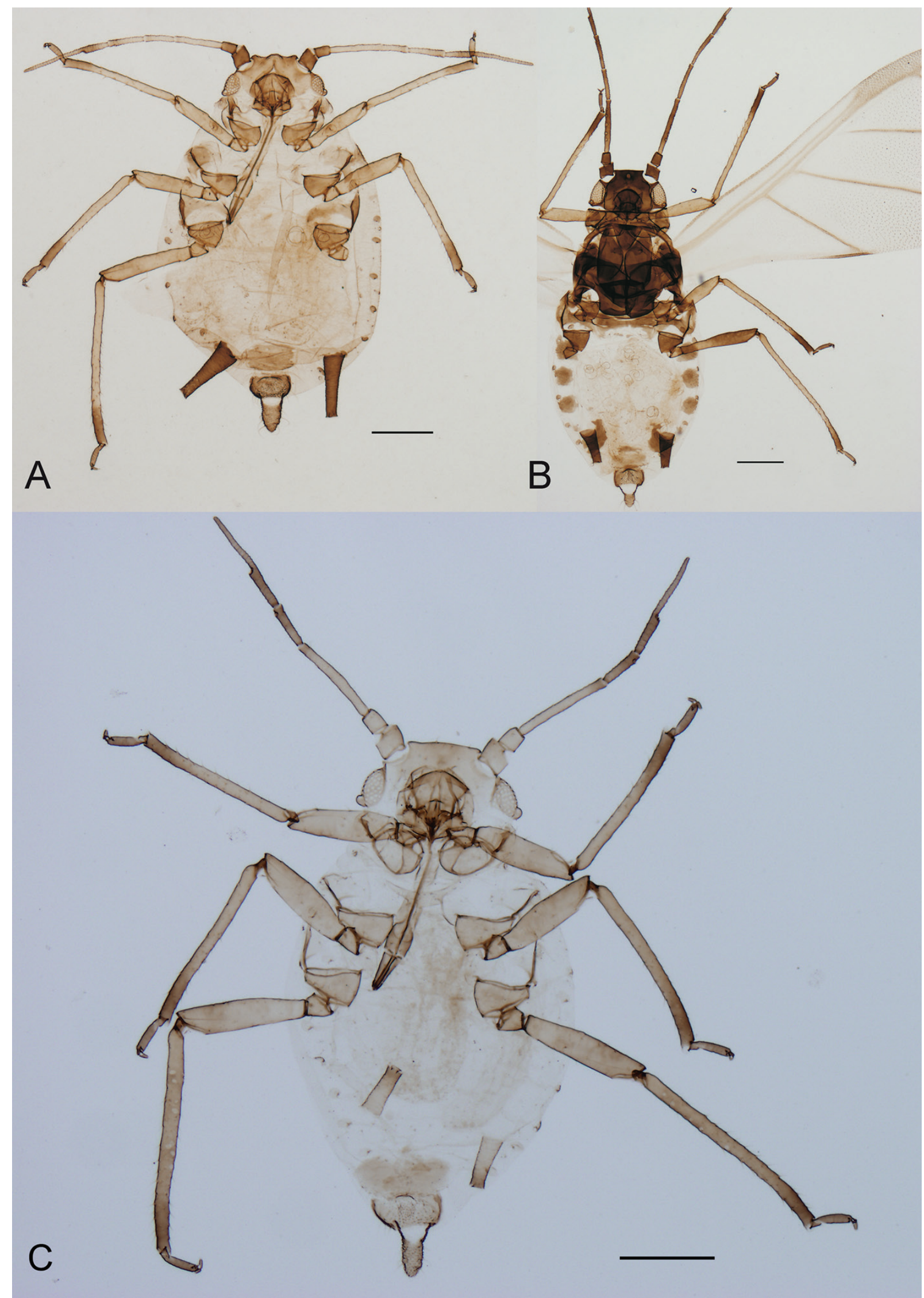

Fig. 3.- A-B, Aphis mulguraeae Nieto Nafría \& Mier Durante, sp. n.; C, Aphis matilei Nieto Nafría, Ortego \& Mier Durante, 2000; $A, C$, hembra vivípara áptera; $B$, hembra vivípara alada. Escalas: $200 \mu \mathrm{m}$.

Fig. 3.- A-B, Aphis mulguraeae Nieto Nafría \& Mier Durante, sp. n.; C, Aphis matilei Nieto Nafría, Ortego \& Mier Durante, 2000 ; A, C, apterous viviparous female; B, alate viviparous female. Scale bars: $200 \mu \mathrm{m}$. 
longitud (su cola es deforme). Otras características métricas en Tabla 1. En vida con cabeza y tórax pardos y abdomen de color verde pálido y cornículos pardo oscuros y cola amarillenta. En preparación la pigmentación de las antenas y de los fémures de las patas medias y posteriores es más intensa y extensa que en las ápteras. Artejo antenal III (de ambas antenas) con 5 sensorios secundarios alineados sobre los 3/4 distales del artejo, redondeados y de tamaño diverso; otros artejos antenales sin ellos. Escleritos intersegmentarios pequeños y tenuemente pigmentados, como los estigmáticos. Segmentos abdominales 1-4 con muy pequeños y dispersos escleritos, segmento 5 con un esclerito espinal y escleritos marginales (poscorniculares) y segmentos 7 y 8 con unas pequeñas bandas transversales. Otras características cualitativas y merísticas como las de las vivíparas ápteras.

\section{Hembras ovíPaRas y Machos: Desconocidos}

Etimología: El nombre específico "junelliae" es el nombre del género al que pertenece la especie de la planta hospedadora de la nueva especie, en genitivo.

Bionomía Y Distribución: Los ejemplares de Aphis junelliae sp. n. fueron recogidos formando un pequeño grupo apretado en ramitas finas de Junellia crithmifolia (Gillies \& Hook. ex Hook.) N. O'Leary \& P. Peralta (Verbenaceae), que es su única planta hospedadora conocida. El ciclo vital es desconocido, pero es posible suponer que sea holocíclico, como en A. matilei.

El género Junellia es sudamericano e incluye una treintena de especies. J. crithmifolia está extendida desde Chubut al sur hasta San Juan al norte (Instituto de Botánica Darwinion, 2017). Cabe la posibilidad de que este pulgón tenga una distribución semejante a la de esa planta, o que incluso sea más extensa si pudiera desarrollarse sobre otras especies del género.

\section{Aphis lippiae Ortego \& Nieto Nafría sp. n.} urn:1sid:zoobank.

org:act:46710295-3639-48F6-AB04-E8818D005E98 Figs. $1 \mathrm{~F}-1 \mathrm{~J}, 2 \mathrm{C}-\mathrm{D}$, Tabla 1

\section{Material tiPo}

Holotipo: hembra vivípara áptera (número 11 de la serie de medidas de la muestra ARG-755), ARGENTINA, provincia de La Rioja, La Rioja [aproximadamente $29^{\circ} 33^{\prime} \mathrm{S}, 66^{\circ} 55^{\prime} \mathrm{W}, 410 \mathrm{~m}$ ], 27-XI2002, sobre Lippia turbinata, Mier Durante, Ortego y Nieto Nafría leg., colección de la Universidad de León (León, España).

Paratipos: 97 hembras vivíparas ápteras [ápt.] y 32 hembras vivíparas aladas [al.], colecciones de la Universidad de León (León, España) y de J. Ortego (Mendoza, Argentina); con los mismos datos que el holotipo (43 ápt. y 16 al.). ARGENTINA, provincia de Córdoba, Villa Dolores, 1-XI-1996 [aproximadamente $31^{\circ} 59^{\prime} \mathrm{S}, 65^{\circ} 13^{\prime} \mathrm{W}, 520 \mathrm{~m}$ ], sobre L. turbinata, Ortego leg. (31 ápt., 15 al.). ARGENTINA, provincia de Mendoza, Malargüe: Borbarán, 23-III-1999 [aproximadamente $36^{\circ} 00^{\prime} \mathrm{S}, 68^{\circ} 26^{\prime} \mathrm{W}, 1430 \mathrm{~m}$ ], sobre L. turbinata, Ortego leg. (23 ápt., 1 al.).

Otros especímenes: ARGENTINA, Mendoza, Termas de Villavicencio (10 km oeste por R.P. 52) [aproximadamente $32^{\circ} 33^{\prime} \mathrm{S}, 68^{\circ} 57^{\prime} \mathrm{W}, 1240 \mathrm{~m}$ ], 30-XI2002, Mier Durante, Ortego y Nieto Nafría leg. (1 ninfa).

Hembras vivíparas ápteras (Figs. 1F, 1I, 1J, 2C): A partir de 98 especímenes (48 medidos). De 1,00 a $1,40 \mathrm{~mm}$ de longitud. Otras características métricas en Tabla 1. En vida de color amarillo verdoso con cornículos pardo oscuros y cola pajiza. Una vez preparados son pálidos en general, con algunas partes del cuerpo más o menos pigmentadas (ver más adelante). Setas del dorso del cuerpo romas, así como las de las antenas, y muchas de las de los fémures. Dorso de la cabeza liso y algo pigmentado. Frente plana. Antenas de seis artejos, en su mayor parte pálidas; buena parte del artejo VI bastante pigmentado (tanto como la parte final de las tibias), artejo I y extremo del artejo V tenuemente pigmentados; artejo III de las antenas con 3-7 setas. Clípeo y láminas mandibulares y maxilares de color pardo, más oscuros que el dorso de la cabeza. El rostro sobrepasa la inserción de las coxas intermedias y puede alcanzar la de las posteriores. Artejo apical relativamente corto y de bordes rectos, de aspecto puntiagudo, ligeramente pigmentado, como el inmediato anterior y el dorso de la cabeza, y con dos setas complementarias más cortas que las primarias. Segmentos torácicos con escleritos marginales de límites muy mal definidos y muy poco pigmentados. Papilas marginales del protórax pálidas y tan voluminosas como el triomatidio. Patas poco o nada pigmentadas salvo los tarsos y el extremo de las tibias, que son las partes del cuerpo más oscuras. Fórmula tarsal 3.3.3. Abdomen sin escleritos segmentarios, escleritos intersegmentarios inapreciables y escleritos estigmáticos muy pequeños y pálidos. Papilas marginales de los segmentos abdominales 1 y 7 algo menos voluminosas que las del protórax y pálidas o muy pálidas. Segmentos abdominales 2-6 sin papilas marginales. Cornículos más o menos tan pigmentados como el clípeo, oscureciéndose ligeramente hacia el ápice, entre subcilíndricos y troncocónicos, con rugosidades transversales marcadas y reborde insignificante. Segmento abdominal 8 con $2-5$ setas. Placas genital y anal muy poco pigmentadas, aquélla con 2 setas discales y 4-10 marginales. Cola corta, con una constricción proximal mínima o sin ella, casi de apariencia triangular, también poco pigmentada; 4-7 setas caudales largas, finas y curvadas. 
Hembras vivíparas aladas (Figs. $1 \mathrm{G}, 1 \mathrm{H}, 2 \mathrm{D}$ ): A partir de 27 especímenes (16 medidos). De 1,31 a 1,64 mm de longitud corporal, incluyendo la cola. Otras características métricas en Tabla 1. En vida con el abdomen de color verde pálido, con cola parduzca y con cabeza, tórax, antenas, final de las patas y cornículos pardos a negros. En preparación los artejos antenales VI (en toda su extensión) y III, IV y V (excepto en una breve porción proximal) con pigmentación similar a la de los tarsos y el final de las tibias. Artejo antenal III a veces fusionado al IV y con 4-12 sensorios secundarios, alineados sobre $3 / 4$ o 4/5 distales del artejo, redondeados y de tamaño variado; artejos IV y V con y $1-4$ y $0-2$ sensorios secundarios, respectivamente, semejantes en forma y algo más pequeños que los del artejo III. Porción distal de los fémures (con menor intensidad los de las patas anteriores) con pigmentación similar a la del final de las tibias. Segmentos 2-5 del abdomen de algunos ejemplares con pequeños y poco pigmentados escleritos marginales; escleritos intersegmentarios inapreciables; escleritos estigmáticos pequeños y poco pigmentados. Segmento abdominal 8 con 3-6 setas. Placa genital con 2-4(5) setas discales y 4-14 marginales. Otras características cualitativas y merísticas como en las ápteras.

\section{Hembras ovíParas y Machos: Desconocidos.}

Eтimología: El nombre específico "lippiae" es el nombre en genitivo del género al que pertenece la planta hospedadora de la nueva especie.

Bionomía y distribución: Aphis lippiae sp. n. forma pequeños grupos en las ramitas finas de Lippia turbinata; su ciclo vital es desconocido, pero es posible suponer que sea holocíclico, como el de A. matilei. El género Lippia tiene varios cientos de especies cuya distribución originaria está limitada a zonas de América del Sur o de África; algo más de veinte de ellas están presentes en Argentina. L. turbinata Griseb está distribuida por gran parte del oeste de la Argentina al norte del río Negro y se conoce también en Chile y Paraguay (Instituto de Botánica Darwinion, 2017). El área de distribución conocida de la nueva especie es la más amplia de las tres nuevas especies, pues se ha colectado en las provincias de Córdoba, Mendoza y La Rioja, y puede que se extienda más al norte sobre esa planta y otras especies de Lippia.

Aphis mulguraeae Nieto Nafría \& Mier Durante, sp. $\mathbf{n}$.

urn:1sid:zoobank.org:act:7A61A543-6314-4361BBCD-EDBF260C23AD

Figs. $1 \mathrm{~K}-1 \mathrm{O}$, 3A-B, Tabla 1

\section{Material tipo}

Holotipo: hembra vivípara áptera (número 9 de la serie de medidas), ARGENTINA, provincia de Mendoza, Luján de Cuyo [aproximadamente $33^{\circ} 05^{\prime} \mathrm{S}$ $68^{\circ} 58^{\prime} \mathrm{W}$ 1050m], 21-XI-2002, sobre Mulguraea aspera, Mier Durante, Nieto Nafría y Ortego leg., colección de la Universidad de León (León, España).

Paratipos: 9 hembras vivíparas ápteras y 4 hembras vivíparas aladas recogidas con el holotipo, colecciones de la Universidad de León (León, España) y de J. Ortego (Mendoza, Argentina).

Hembras Vivíparas ápteras (Figs. $1 \mathrm{~K}, 1 \mathrm{~N}$, 1O, 3A): A partir de 10 especímenes. De 1,02 a 1,30 $\mathrm{mm}$ de longitud del cuerpo, incluyendo la cola. Otras características métricas en Tabla 1. En vida de color amarillo pajizo o verde con manchas amarillas o rosadas, con cola pajiza y cornículos casi negros. Una vez preparados son pálidos en general, con algunas partes del cuerpo más o menos pigmentadas (ver más adelante). Setas del dorso del cuerpo, de antenas y de patas finas y apuntadas. Frente sinuosa, con tubérculo medial marcado. Dorso de la cabeza algo pigmentado, habitualmente menos que el clípeo y el artejo antenal I. Artejos antenales II, VI y extremo de III, IV y V tenuemente pigmentados. Artejo antenal III con 2-4 setas. Rostro largo (alcanza la inserción de las coxas de las patas posteriores). Artejo apical del rostro alargado y con bordes casi rectilíneos, ligeramente pigmentado (como el penúltimo) y con dos setas complementarias muy finas y tan largas como las primarias. Segmentos torácicos con escleritos marginales de límites muy mal definidos y muy poco pigmentados. Papilas marginales del protórax casi tan anchas como el triomatidio, pero muy poco elevadas, pálidas. Patas pálidas en su mayor parte, con tarsos y porción final de las tibias pigmentados. Fórmula tarsal 3.3.3. Abdomen sin escleritos segmentarios, escleritos intersegmentarios inapreciables, escleritos estigmáticos pequeños y tenuemente pigmentados. Papilas marginales de los segmentos abdominales 1 y 7 pequeñas, menos voluminosas que las del protórax (difíciles de apreciar en algunos ejemplares). Segmentos intermedios del abdomen sin papilas marginales. Cornículos oscuros o muy oscuros, robustos, troncocónicos, con rugosidades transversales, y con un reborde insignificante. Segmento abdominal 8 con $2-3$ setas. Placa genital pálida con 2 setas discales y 5-8 marginales. Placa anal algo pigmentada. Cola robusta, con constricción muy poco marcada y con pigmentación similar a la de la placa anal y mucho menor que la de los cornículos; con 4-7 setas largas, finas y curvadas.

Hembras vivíparas aladas (Figs. 1L, 1M, 3B): A partir de 4 especímenes. De 1,20 a 1,40 mm de longitud. Otras características métricas en Tabla 1. En vida con cabeza, tórax y cornículos negros y con 
abdomen de color verde pálido con manchas marginales pardas y cola pajiza. En preparación, antenas y fémures de las patas posteriores más extensamente pigmentados que los de las ápteras. Escleritos marginales bien pigmentados en los segmentos abdominales 1 (pequeños), 2-4 (grandes), 5 (precorniculares, pequeños) y 6 (postcorniculares, grandes); segmentos precorniculares con esclerotización espinal variada, nula en dos ejemplares y con algunos escleritos dispersos, pequeños y de contorno poligonal en otros dos ejemplares; segmentos 6-8 con bandas transversales pequeñas; escleritos intersegmentarios pequeños y tenuemente pigmentados o inapreciables; escleritos estigmáticos pequeños y poco pigmentados. Artejo antenal III con 4-6 setas y también con 4-6 sensorios secundarios redondeados, bastante grandes, y alineados sobre casi toda la longitud del artejo; otros artejos antenales sin ellos. Otras características cualitativas y merísticas como en las ápteras.

\section{Hembras ovíparas y machos: Desconocidos.}

Etimología: El nombre específico "mulguraeae" es el nombre en genitivo del género de su planta hospedadora.

Bionomía y Distribución: Mulguraea aspera (Gillies \& Hook.) N. O'Leary \& P. Peralta (Verbenaceae) es la única planta hospedadora conocida para Aphis mulguraeae sp. n.; los ejemplares recogidos se encontraban dispersos en ramitas finas. El ciclo vital es desconocido, pero es posible suponer que sea holocíclico, como en A. matilei. El género Mulguraea se ha constituido recientemente con 13 especies sudamericanas (O'Leary et al., 2009). M. aspera se encuentra distribuida entre Río Negro al sur y Catamarca al norte (Instituto de Botánica Darwinion, 2017). Esta nueva especie se conoce solamente de una localidad de Mendoza, aunque su distribución puede ser más extensa, como se ha señalado en las otras nuevas especies.

\section{Discusión taxonómica}

Las hembras vivíparas ápteras de las tres nuevas especies se pueden diferenciar entre sí por un grupo de caracteres cualitativos y algunos caracteres métricos: forma y robustez de los cornículos, forma de la frente, forma de la cola, forma del artejo apical del rostro, pigmentación de la placa genital, pigmentación de patas y antenas, proporción entre el filamento terminal y la base del artejo antenal VI, proporción entre el artejo apical del rostro y el $2 .^{\circ}$ de los tarsos posteriores, y medidas absolutas y relativas de los cornículos (Figs. 1, 2, 3, Tabla 1).

Las hembras vivíparas ápteras de las tres nuevas especies se parecen a $A$. matilei (Figs. 1P, 1Q, 1R), la otra especie sudamericana de Aphis cuya planta hospedadora también es una verbenácea (Fig. 7), entre otras características: I) por el cuerpo pálido en preparación microscópica con ausencia de escleritos segmentarios por delante de los cornículos, salvo en algunos ejemplares de $A$. junelliae sp. n., que presentan algunos espinales, y II) por el inapreciable reborde apical de los cornículos. Se diferencian: a) por la pigmentación de los cornículos, que en las nuevas especies están bien pigmentados mientras que en A. matilei son pálidos, al menos en su mitad proximal y se van oscureciendo hacia el ápice en la distal, y b) por la ausencia de papilas marginales en el segmento 7 del abdomen en la mayor parte de los ejemplares de A. matilei (ver Nieto Nafría et al., 2000), papilas que se encuentran siempre presentes en los especímenes de las tres nuevas especies.

Solamente las hembras vivíparas ápteras de cuatro especies de las 56 de Aphis citadas hasta ahora en América del Sur comparten las siguientes cinco características con las tres nuevas especies (Nieto Nafría et al., 2016; López Ciruelos et al., 2017): (1) presencia de papilas marginales en los segmentos abdominales 1 y 7, (2) ausencia de papilas marginales en los segmentos abdominales intermedios, (3) cornículos oscuros, al menos tan pigmentados como la parte final de las tibias, (4) fémures o buena parte de ellos tan pálidos como la mayor parte de las tibias, (5) esclerotización espinal o marginal en los segmentos abdominales precorniculares ausente o con presencia muy limitada. Son las americanas A. amaranthi Holman, 1974, A. martinezi Nieto Nafría, Ortego \& Mier Durante, 1999 y las introducidas A. gossypii Glover, 1877 y $A$. sedi Kaltenbach, 1843. A. amaranthi fue descrita de Cuba y en América del Sur se conoce en Argentina y Brasil, vive en la parte basal de plantas de especies de Amaranthus (Amaranthaceae). A. martinezi se conoce solamente en Argentina sobre plantas del género Mulinum. A. gossypii es polífaga (entre sus plantas hospedadoras reconocidas hay verbenáceas) y ha devenido cosmopolita. A. sedi vive fundamentalmente sobre crasuláceas y presenta una amplia distribución por acción antrópica, con presencia reconocida en Argentina y Chile.

Esas cuatro especies se pueden diferenciar de A. junelliae sp. n., de A. lippiae sp. n. y de A. mulguraeae sp. n. atendiendo a los siguientes caracteres morfológicos a partir de los datos aportados por Blackman \& Eastop (2017), García Prieto \& Nieto Nafría (2005), González Rodríguez et al, (2017), Holman (1974) y López Ciruelos et al. (2017), y la observación de ejemplares de la colección afidológica de la Universidad de León. En muchos ejemplares de A. amaranthi los fémures suelen estar pigmentados al menos en su mitad distal, es frecuente la presencia de papilas marginales en segmentos intermedios del abdomen, la proporción entre el filamento terminal y la base del artejo antenal VI es diferente a la 
de las nuevas especies (2,2-2,7 veces, frente a 1,92 como máximo en $A$. junelliae sp. n. y $A$. lippiae sp. n. y 2,6 como mínimo en $A$. mulguraeae sp. n.). En A. martinezi los cornículos son tan largos como la cola como máximo; en A. junelliae sp. n. los cornículos son también cortos con relación a la cola, pero ésta es digitiforme (con poca constricción proximal) y aquéllos no están curvados hacia el exterior, mientras que en A. martinezi la cola es triangular ( $\sin$ constricción proximal) y los cornículos están curvados hacia el exterior. La forma de la cola y sus medidas en relación con los cornículos permiten separar A. lippiae sp. n. y A. mulguraeae sp. n. de $A$. gossypii y de $A$. sedi. Éstas dos especies se pueden separar de A. junelliae sp. $\mathbf{n}$. por el aspecto y longitud de los cornículos $(0,17-0,34$ y $0,14-0,23 \mathrm{~mm}$ respectivamente en $A$. gossypii y $A$. sedi, $0,10-0,12 \mathrm{~mm}$ en $A$. junelliae sp. n.) y por la proporción entre el filamento terminal y la base del artejo antenal VI $(2,0-3,5$ y $1,5-2,3$ veces respectivamente en $A$. gossypii y $A$. sedi, $1,1-1,7 \mathrm{~mm}$ en $A$. junelliae sp. n.).

\section{Agradecimientos}

Los autores agradecen la información sobre verbenáceas en Argentina facilitada por la Prof. ${ }^{a}$ María E. Múlgura de Romero [Instituto de Botánica Darwinion (CONYCET), Acassuso (Buenos Aires, Argentina)], así como la contribución de Sara I. López Ciruelos [Servicio de Microscopía, Universidad de León] en la realización de las microfotografías.

\section{Referencias}

Barbagallo, S. \& Massimino Cocuzza, G. E., 2014. A survey of the aphid fauna in the Italian regions of Latium and Campania. Redia, 97: 19-47.

Blackman, R. L. \& Eastop, V. F, 2017. Aphids on the World's plants. An online identification and information guide. Disponible en: http:/www.aphidsonworldsplants.info/ (consulta en octubre de 2017).

Favret, C., 2017. Aphids Species File. Version 5.0/5.0. Disponible en: http://Aphid.SpeciesFile.org (consulta en octubre de 2017)
García Prieto, F. \& Nieto Nafría, J. M., 2005. Género Aphis Linnaeus, 1758. In: J. M. Nieto Nafría, M. P. Mier Durante, F. García Prieto \& N. Pérez Hidalgo. Hemiptera Aphididae III. In: M. A. Ramos et al. (eds.). Fauna Ibérica, vol. 28. Museo Nacional de Ciencias Naturales (CSIC), Madrid. España: 30-174.

González Rodríguez, S., Brown, P. A., Ortego, J., López Ciruelos, S. I. \& Nieto Nafría, J. M., 2017. Aphis species (Hemiptera, Aphididae) living on Mulinum (Apiaceae) in South America, with a description of a new species. Zootaxa, 4216 (1): 47-54.https://doi.org/10.11646/zootaxa.4216.1.2

Holman, J., 1974. Los áfidos de Cuba. Instituto Cubano del Libro. La Habana. 304 pp.

Instituto de Botánica Darwinion, 2017. Flora del cono Sur. Disponible en: http://www.darwin.edu.ar/proyectos/ floraargentina/fa.htm (consulta en octubre de 2017).

López Ciruelos, S. I., Ortego, J., Mier Durante, M. P. \& Nieto Nafría, J. M., 2017 A new species of Aphis (Hem. Aphididae) collected on Gymnophyton (Apiaceae) in Argentina Graellsia, 73(1): e055 (9 pp.). https://doi. org/10.3989/graellsia.2017.v73.171

Múlgura de Romero, M. E., Rotman A. D., Atkins, S., 2012. Verbenaceae Adans. In: Flora Argentina. Disponible en http://www.floraargentina.edu.ar/publicaciones/ VERBENACEAE.pdf (consulta septiembre de 2017).

Nieto Nafría, J. M., Fuentes-Contreras, E., Castro Colmenero, M., Aldea Piera, M., Ortego J. \& Mier Durante M. P., 2016. Catálogo de los áfidos (Hemiptera, Aphididae) de Chile, con plantas hospedadoras y distribuciones regional y provincial. Graellsia, 72(2): e050 (35 pp.). https://doi.org/10.3989/graellsia.2016.v72.167

Nieto Nafría, J. M. \& Mier Durante, M. P., 1998. Hemiptera Aphididae I. In: M.A. Ramos et al. (eds.). Fauna Ibérica, vol. 11. Museo Nacional de Ciencias Naturales (CSIC). Madrid. 424 pp.

Nieto Nafría, J. M., Ortego, J. \& Mier Durante, M. P., 2000. Una nueva especie argentina del género Aphis, propia de Verbena [Hemiptera, Aphididae; Verbenaceae]. Revue Française d'Entomologie (N.S.), 22(4): 173-176.

O’Leary, N., Yuan Y.-W., Chemisquy, A. \& Olmstead, R. G., 2009. Reassignment of species of paraphyletic Junellia s. 1. to the new genus Mulguraea (Verbenaceae) and new circumscription of genus Junellia: molecular and morphological congruence. Systematic Botany, 34(4): 777-786. https://doi.org/10.1600/036364409790139691

The Plant List, 2013. The Plant List. Version 1.1. Disponible en: http://www.theplantlist.org/ (consulta en octubre de 2017). 